\title{
Consumer Handwashing Research: Handwashing in a Pandemic
}

Area of research interest: Behaviour and perception

Planned completion: 25 August 2022

Project status: Completed

Authors: Helen Heard

Conducted by: Food Standards Agency and Ipsos

Date published: 15 October 2021

\section{Background}

Between April 2020 and January 2022, the Food Standards Agency (FSA) with Ipsos collected data on handwashing to understand how and why people wash their hands and the impact the pandemic has had on their handwashing behaviour.

Two reports are available on this topic.

1) The 'Consumer Handwashing in a Pandemic Research' report (and associated Appendices) was published in October 2021. This report presents findings from the qualitative research conducted by the FSA alongside other literature available on the topic of hand hygiene to provide a comprehensive overview of consumer handwashing behaviour during the pandemic. This report also includes the first four waves of the quarterly quantitative handwashing tracker survey (April 2020 - January 2021).

2) The 'Consumer Handwashing tracker; Key findings from April 2020-January 2022' was published in August 2022. This report provides the latest insights from the quarterly quantitative handwashing tracker survey (April 2020 - January 2022). This is the final report from this survey, and comments on key trends overtime since tracking began. 